\title{
Introduction
}

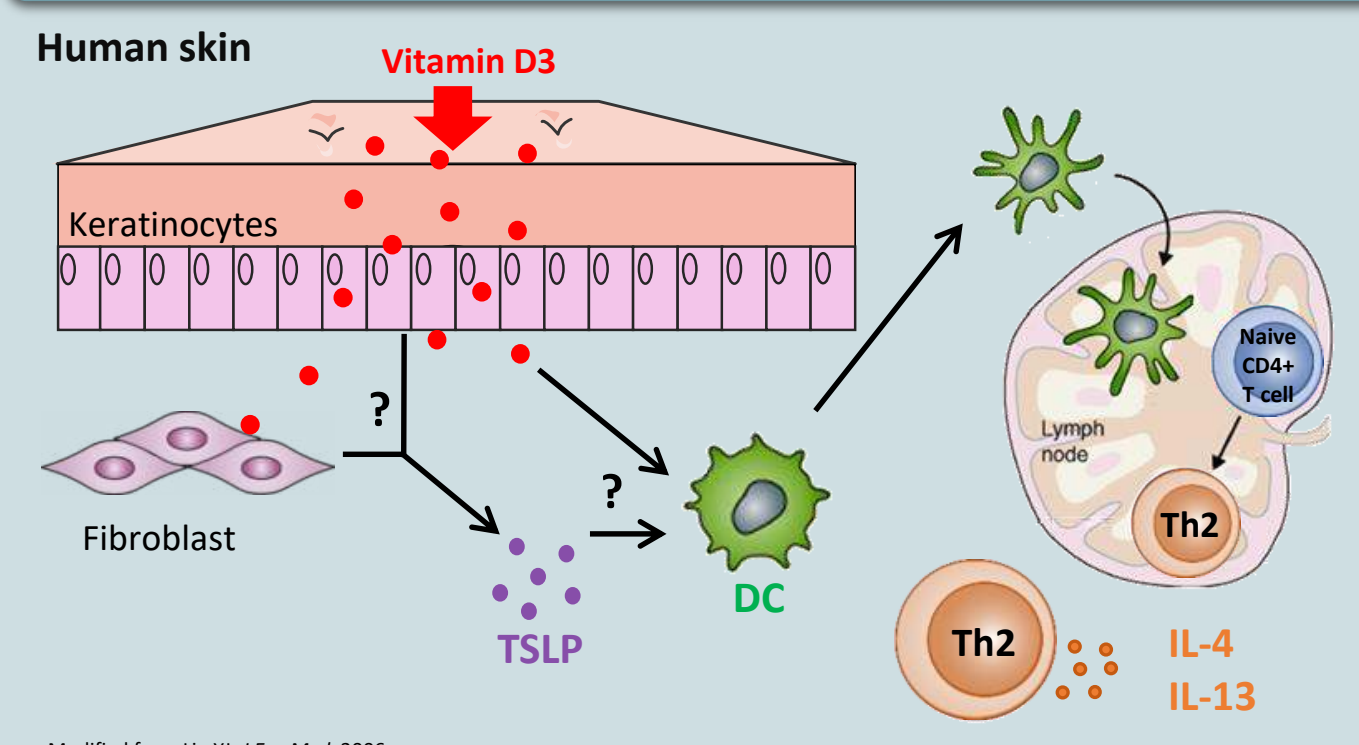

The vitamin D3 (cholecalciferol) is a steroid hormone that controls calcium and phosphate metabolism, but it also regulates other essential pathways. Indeed, vitamin D3 affects multiple functions in the skin such as keratinocyte homeostasis, barrier maintenance and immunoregulatory processes (1). A major mechanism by which vitamin D3 modulates immune responses is the regulation of dendritic cells (DCs) (2). Previous publications propose that human DCs treated with vitamin D3 acquire an antiinflammatory capacity, either by promoting Treg differentiation (3) or by counteracting $T$ helper 1 (Th1)/Th17 polarisation (4). In mice, Thymic Stromal Lymphopoietin (TSLP) production is induced in keratinocytes and fibroblasts by topical application of vitamin D3 (5), but it remains unclear whether a similar effect can be achieved in humans. In both species, however, TSLP-exposed DCs prime for Th2 differentiation (6). Our goal is to study the effect of two vitamin D3 analogs (calcitriol, calcipotriol) on TSLP expression in normal human skin and to clarify whether vitamin D3 promotes human Th2 responses by TSLP-dependent or TSLP-independent pathways.

\section{Experimental protocol}

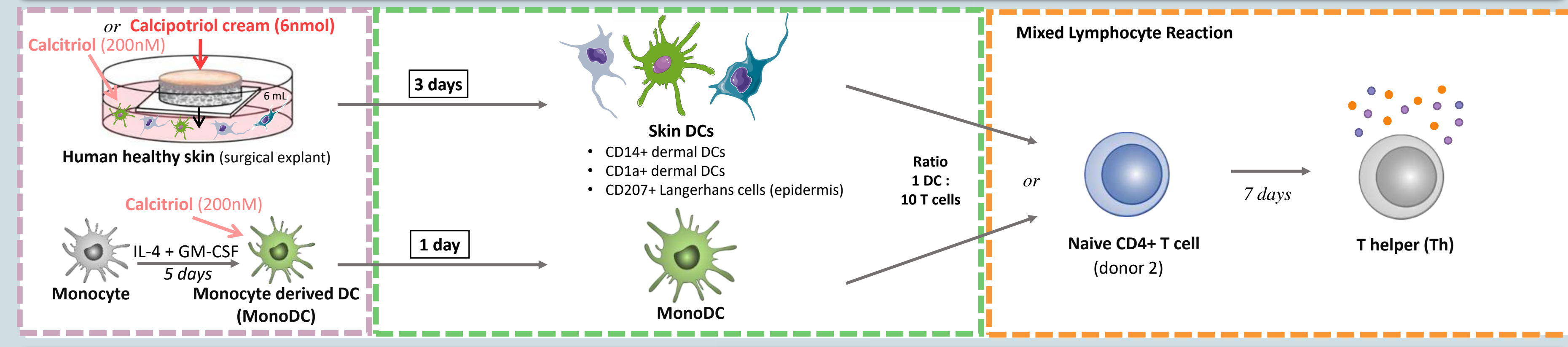

Results

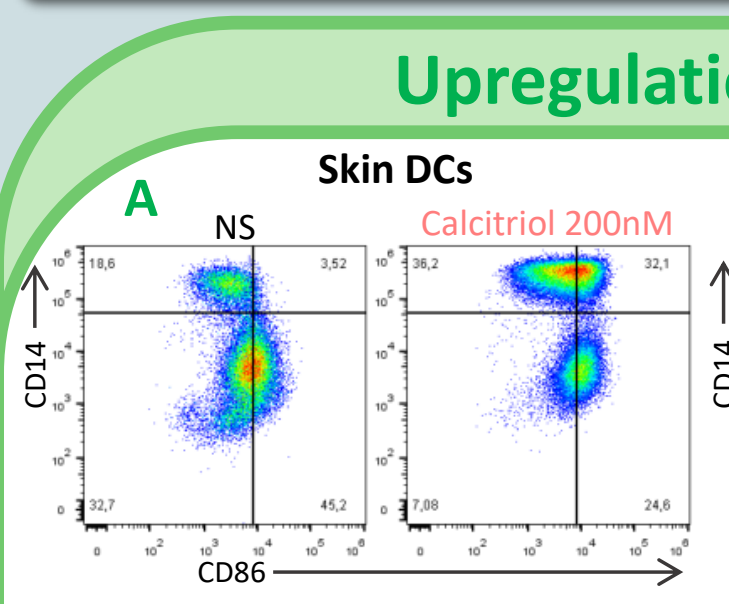

C

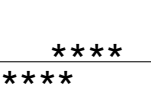
$\stackrel{* * * *}{* * * \star}$

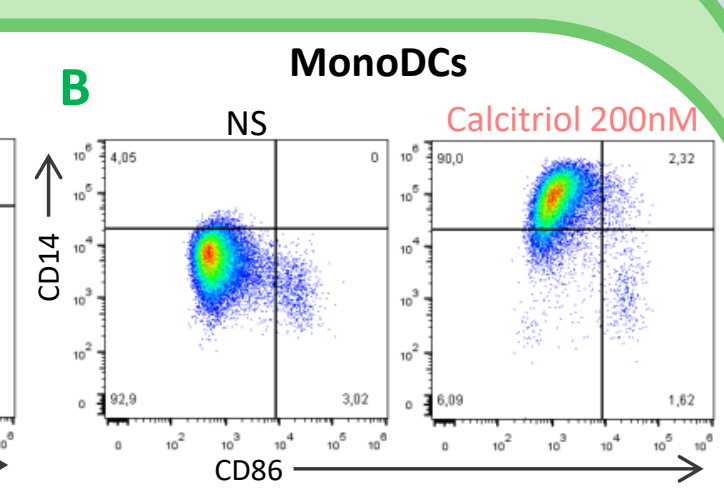

D
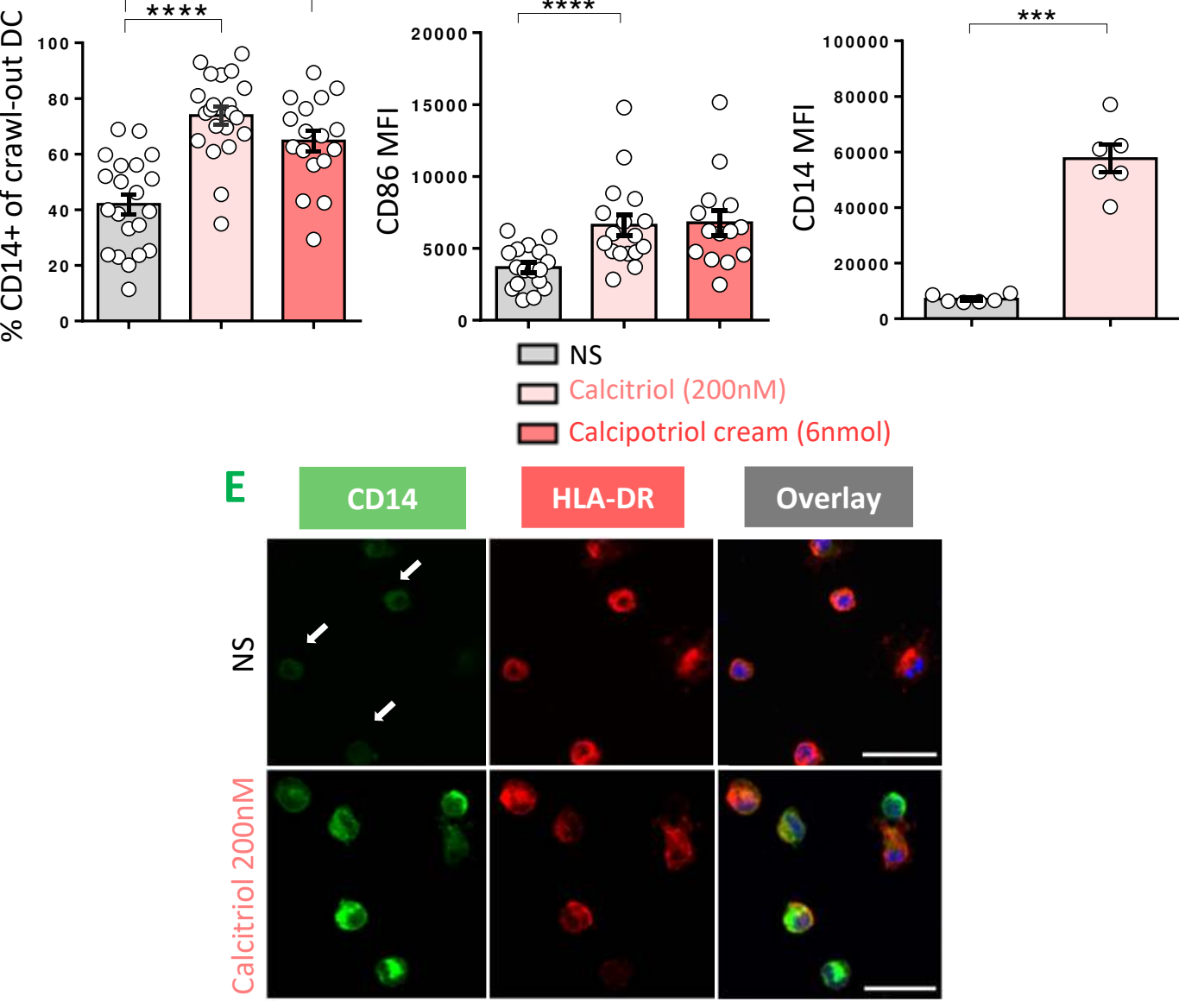

Figure 1 : Vitamin D3 analogs promote upregulation of CD14. Flow cytometry analysis of CD14 and CD86 expression on total skin DCs (A-C) and MonoDCs (B-D). Datas are presented as Mean \pm SEM. Paired t-test. ${ }^{* * *} \mathrm{p}<$ $0.001, * * * * \mathrm{p}<0.0001$ Immunofluorescence staining on skin DCs (E). Scale bar $=25 \mu \mathrm{M}$.
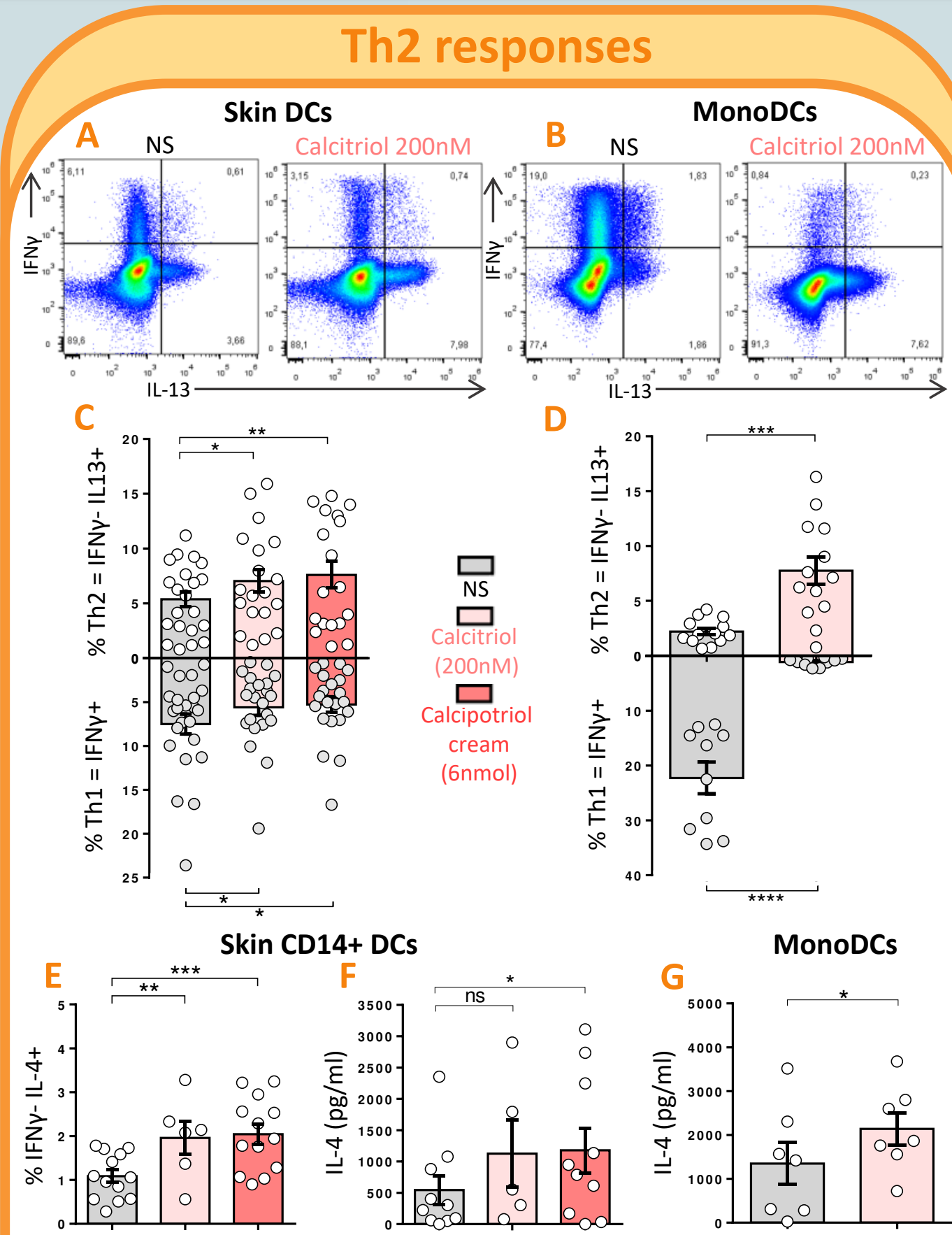

Figure 2 : Vitamin D3-stimulated DCs promote human Th2 responses. Analysis of \%Th2 (IFNY- IL-13+ or IL-4+) and \%Th1 (IFN $\left.{ }^{+}\right)$after coculture with vitamin D3-stimulated total skin DCs (A-C), MonoDCs (B-D), CD14+ DCs (E). IL-4 in 24h-supernatants of $\mathrm{T}$ cells restimulated by PMA/ionomycin $\left(\right.$ F-G). Paired t-test. ns: not significant, ${ }^{*} \mathrm{p}<$ ${ }^{* *} \mathrm{p}<0.001,{ }^{* * *} \mathrm{p}<0.001{ }^{* * * *} \mathrm{p}<0.0001$.

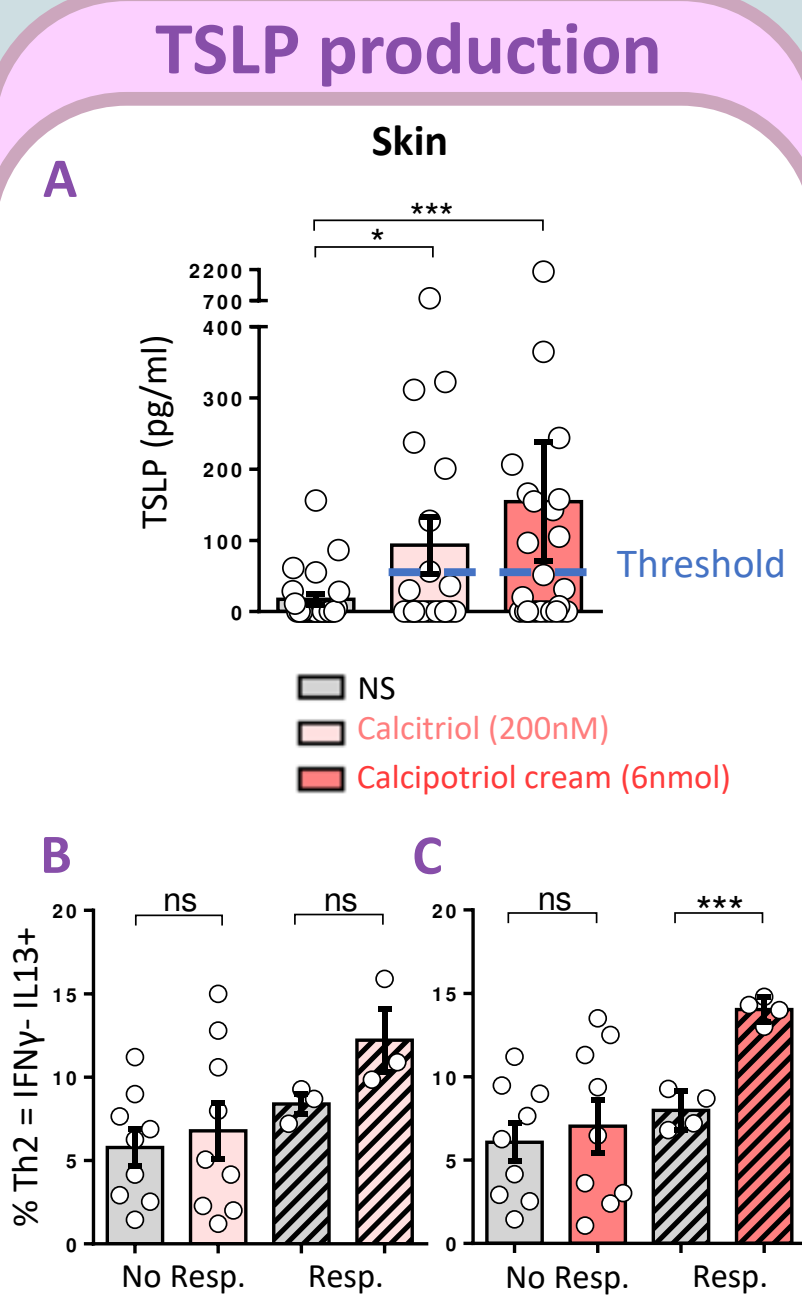

Figure 3 : Vitamin D3 induces TSLP protein expression in human skin biopsies.

After 3 days of stimulation, skin supernatant is collected and analyzed for TSLP expression by ELISA $(n=30)$ (A). Percentage of IFNY- IL-13+ is represented in responsive (TSLP > Mean-SEM) (B) and no responsive (TSLP $<$ MeanSEM) (C) donors. Datas are presented as Mean \pm SEM. Paired $t$ tests. ns: not significant, ${ }^{*} p<0.05$, $* * * p<0.001$.

\section{Bibliography}

(1) Holick MF. J Cell Biochem. 2003

(2) Bscheider M. Immunology. 2016.

(3) Bakdash G. et al. Hum Vaccin Immunother. 2013.

(4) Daniel C. et al J Pharmacol Exp Ther. 2007 (5) Li M. et al. Proc Natl Acad Sci U S A. 2006 (6) Ito T. et al. J. Exp. Med 2005.
We show that vitamin D3 induces TSLP production by healthy human skin. Moreover, we demonstrate an upregulation of CD14
by all DCs exposed to vitamin D3, since the direct effect on MonoDCs does not support an increased migration of CD14+ dermal DCs. These vitamin D3-stimulated DCs promote human Th2 responses. Although a direct link has been established between TSLP and Th2 polarization, the strong Th2 response induced by vitamin D3-stimulated MonoDCs also suggests a TSLP-independent effect. We will further investigate this by interfering with TSLP and vitamin D3 signaling pathways. Next, we intend to use vitamin D3 to reproduce early atopic dermatitis features using a novel innervated and immunocompetent in vitro human skin model. 